\title{
Symbols and Hold-up
}

\author{
H. Morita ${ }^{a}$ and M. Servátka ${ }^{b}$ \\ ${ }^{a}$ School of Economics, Australian School of Business, University of New South Wales \\ ${ }^{b}$ New Zealand Experimental Economics Laboratory, Department of Economics and Finance, University of \\ Canterbury \\ Email: maros.servatka@,canterbury.ac.nz
}

\begin{abstract}
:
Social psychology research shows that symbols play an important role in reinforcing group identity and enhancing cooperation among ingroup members by differentiating them from outgroup members. Symbols provide a clear way of identifying group boundaries and thus allow for achieving the benefits of cooperation without the risk of excessive costs by limiting altruistic behavior towards ingroup members. Social psychologists describe ingroup as a bounded community of mutual and depersonalized expectations of cooperation. Such expectations motivate adherence to ingroup norms and promote behavior that ensures that one is recognized as an ingroup member. This causes the use of symbols in the field being often confounded with communication, social interaction and possibly other contributing factors, making it hard to clearly identify their contribution to creating identity and subsequent effect on strategic decision-making, thus opening the door for their experimental separation. In this paper we present an experiment that separates the roles of symbols from helping behavior in creating group identity which is sufficiently strong to mitigate the hold-up problem.
\end{abstract}

Keywords: altruism, experiment, hold-up problem, group identity, integration, other-regarding preferences, relation-specific investment, team membership 


\section{INTRODUCTION}

Social psychology research shows that symbols play an important role in reinforcing group identity and enhancing cooperation among ingroup members by differentiating them from outgroup members. Symbols provide a clear way of identifying group boundaries and thus allow for achieving the benefits of cooperation without the risk of excessive costs by limiting altruistic behavior towards ingroup members. Social psychologists describe ingroup as a bounded community of mutual and depersonalized expectations of cooperation. Such expectations motivate adherence to ingroup norms and promote behavior that ensures that one is recognized as an ingroup member (Brewer, 1981 and 1999). This causes the use of symbols in the field being often confounded with communication, social interaction and possibly other contributing factors, making it hard to clearly identify their contribution to creating identity and subsequent effect on strategic decision-making, thus opening the door for their experimental separation.

One particular area where group identity has been shown to override strong economic incentives is the holdup problem. Under the standard setup, relation-specific investment in bilateral trade creates a surplus to be shared between two parties because the value of such investment is appreciably lower in any use other than supporting the transaction between the two parties. In a world of incomplete contracts and self-regarding preferences the surplus-sharing leads to inefficiency due to the lack of investment. Morita and Servátka (2011, henceforth MS) experimentally demonstrate that group identity is capable of mitigating hold-up. In the experiment the subjects were randomly divided into two teams, identified by team symbols (different color t-shirts). Prior to making decisions in the hold-up game the members of the same team could communicate with one another via an online chat and provide/receive help to answer trivia questions. The team membership was thus induced by team symbols as well as team members' helping behavior. MS observe that when the subjects were paired with another team-member, their altruism increased compared to a situation when they are paired with a member of the other team. As a result, they were more likely to invest and share the surplus following an investment. While such design provides evidence that group identity can alter economic incentives, it does not allow for a conclusion whether symbols themselves (through identifying group boundaries) are sufficient to induce group identity and influence decisions. In this paper we present an experiment that separates the roles of symbols from helping behavior in creating group identity which is sufficiently strong to mitigate the hold-up problem.

\section{EXPERIMENTAL DESIGN AND PROCEDURES}

In the experiment the social identity is created solely with the use of symbols. The experiment included two treatments based on pairing of subjects (Same-Team and Different-Team) implemented in an across-subjects design. Below we summarize the experimental protocol.

At the beginning of the experiment the participants were randomly divided into Orange Team and Yellow Team by drawing colored pieces of paper from a large opaque envelope. Orange Team was seated in the front of the room and Yellow Team in the back. The experimenters then handed subjects their respective color t-shirts, representing team uniforms, and asked everyone to put them on. The subjects were told they could keep their t-shirts after the experiment was over. Once everyone was wearing a t-shirt, we sequentially asked the teams to get up, look at their teammates and verify that everyone on the team was wearing the same color.

Then neutrally framed instructions were handed out. In the Same-Team treatment, the subjects were instructed that each person from the Yellow Team would be randomly paired with another person from the Yellow Team and each person from the Orange Team with another person from the Orange Team. In the Different-Team treatment, the subjects were instructed that each person from the Yellow Team would be randomly paired with a person from the Orange Team. The instructions emphasized that the interaction was anonymous and that the experimenters would keep track of all decisions using ID numbers.

A coin was publicly flipped to randomly determine the roles depending on the row in which a subject was sitting. The allocation of the First Mover (henceforth FM) and Second Mover (SM) to a particular pair was done by experimenter based on a pre-assigned matching, unknown to the subjects. The decisions were divided into three stages. In Stage 1, the FM had to decide whether or not to invest his/her 10 NZD show up fee in order to create 14 NZD for the pair. If the FM decided not to invest the 10 NZD show up fee, then no money was created and both movers kept their show up fees. If the FM decided to invest, then 14 NZD was made available to split between the two paired persons. An offer of how to split the 14 NZD was determined by the SM in Stage 2. In Stage 3, the FM learned about the offer, and could either accept it or reject it. If the FM accepted the offer, both movers received the respective amounts stated in the offer. If the FM rejected, 
the 14 NZD disappeared and both the FM and the SM received 0 NZD. (The SM still kept the show up fee of 10 NZD.)

In an attempt to minimize confusion of subjects we included three control questions which all participants had to answer correctly before proceeding to the decision-making part. The SM's offers for the control questions were generated randomly for each session. After the subjects answered the questions, the experimenters verified their correctness by inspecting each subject's answers individually and if necessary, provided additional assistance and explanation until the subject calculated all answers correctly. Then the three scenarios were summarized publicly by the experimenter.

Before the decision-making part started, the subjects were reminded about their pairing. In order to transfer information between matched pairs, one of the experimenters collected and later redistributed all decision sheets, while the second experimenter copied the decisions from one sheet to another. This procedure was implemented with the aim to prevent the exchange of superfluous information during the game and aid in maintaining the anonymity of individual decisions.

At the end of the session we asked subjects to complete a short post-experiment questionnaire and offered 5 NZD for doing so to ensure that no subject left the experiment with zero payoffs. Finally, all subjects were privately paid their earnings for the session.

\section{RESULTS}

The experiment took place in the New Zealand Experimental Economics Laboratory (NZEEL) at the University of Canterbury with undergraduate students serving as subjects. The recruited subjects have never previously participated in an economic experiment at this university. On average, an experimental session lasted around 60 minutes including initial instruction period and payment of subjects.

Fifty-four subject pairs participated in the Same-Team treatment. In Stage 1, twenty FMs invested, yielding an investment rate of 37\%. Following an investment SMs offered on average 8.55 NZD in Stage 2. Only one of these offers (6 NZD) was rejected in Stage 3 by the respective FM, resulting in a rejection rate of $5 \%$.

Sixty-two subject pairs participated in the Different-Team treatment. In Stage 1, sixteen FMs invested, yielding an investment rate of 25.8\%. Following an investment SMs offered on average 7.75 NZD in Stage 2. Two of these offers (both 4 NZD) were rejected in Stage 3 by the respective FM, resulting in a rejection rate of $12.5 \%$.

First, we test whether the group identity created by t-shirts was strong enough to induce FMs to invest more often when paired with their team members as opposed to members of the other team. We compare the FMs' investment rates in our two treatments: While the investment rate in the Same-Team treatment is higher than in the Different-Team treatment, the one-sided Fisher's exact test reveals that the difference is marginally insignificant $(p=0.135)$.

Second, we compare whether upon the FM's investment, the SM's offer is higher in the presence of group identity induced by the use of symbols. As suggested by HS the reason behind such difference would be due to a higher level of SM's altruism induced by group identity. The one-sided Wilcoxon rank-sum test detects that the offers in the Same-Team treatment were no different than in the Different-Team treatment $(p=0.282)$. In both treatments those FMs who invested lost money. This result suggests that group identity induced by tshirts was not strong enough to sufficiently strengthen agents' other-regarding preferences to mitigate holdup. Finally, due to a small number of observations, we are unable to meaningfully compare the rejections rates in Stage 3.

\section{DISCUSSION AND CONCLUSIONS}

We show that group identity has a potential to mitigate the hold-up problem by strengthening agents' altruistic preferences. However, as with most (if not all) experiments studying social phenomena in laboratory conditions, one has to be cautious when interpreting the results as they may vary in different strategic and contextual environments (Levitt and List, 2007). It is also possible that a different nature and level of scrutiny might influence the behavior in other settings.

\section{ACKNOWLEDGMENTS}

Financial support was provided by the University of Canterbury, College of Business and Economics. 


\section{REFERENCES}

Levitt, S. and J. List (2007) "What Do Laboratory Experiments Measuring Social Preferences Tell Us about the Real World," Journal of Economic Perspectives, 2007, 21(2), 153-174.

Morita, H. and Servátka, M. (2011). Group Identity and Relation-Specific Investment: An Experimental Investigation. University of Canterbury working paper. 